\title{
Aspergillus fumigatus colonisation and population density of place of residence in cystic fibrosis
}

\author{
E J Simmonds, J M Littlewood, V Hopwood, E G V Evans
}

\begin{abstract}
The relation between antibody titres of aspergillus in patients with cystic fibrosis and the population density of their place of residence was investigated. Patients with high titres of antibodies to Aspergillus fumigatus were significantly more likely to live in an area of low population density. Living in a rural environment may predispose to $A$ fumigatus colonisation.
\end{abstract}

(Arch Dis Child 1994; 70: 139-140)

Colonisation of the respiratory tract with Aspergillus fumigatus is common in patients with cystic fibrosis. The factors that lead to this phenomenon are not clear, though it is recognised that patients in a poorer clinical state have higher levels of antibodies. ${ }^{1}$ The degree of exposure to the fungus may also be important, though this has not been studied previously. As high spore concentrations are found near rotting vegetation, compost, and animal houses, ${ }^{2}$ we speculated that colonisation with $A$ fumigatus might be associated with living in a rural area. We chose to investigate this by comparing the population density of patients' home addresses with the titre of antibody to aspergillus.

\section{Patients and methods}

PATIENTS

The study population consisted of the 137 patients who attended the Leeds Regional Cystic Fibrosis Unit for regular follow up studies at the end of 1988 . For each of the patients the titres of antibody to aspergillus and the population density of the electoral ward inhabited were analysed in a cross sectional study.

Regional Cystic

Fibrosis Unit, St

James's University

Hospital, Leeds

E J Simmonds

J M Littlewood

PHLS Mycology

Reference Laboratory,

Department of

Microbiology,

University of Leeds

and General Infirmary

V Hopwood

E G V Evan

Correspondence to:

Dr E J Simmonds,

Department of Child Health,

Leicester Royal Infirmary,

Leicester LE1 5WW.

Accepted 14 September

1993

MEASUREMENT OF ANTIBODY TO ASPERGILLUS measured using a previously described enzyme linked immunosorbent assay (ELISA) to detect antiaspergillus IgG. ${ }^{3}$ A single serum sample was assayed in triplicate for each patient. The absorbance (A) of the samples was read in an ELISA reader at 492 and 405 $\mathrm{nm}$. The results were expressed as a specific binding index (SBI) using the formula

$$
\mathrm{SBI} \%=\frac{\mathrm{A}_{\text {sample }}-\mathrm{A}_{\text {negative control }}}{\mathrm{A}_{\text {positive control }}-\mathrm{A}_{\text {negative control }}}
$$

The SBI for each wavelength was averaged to
POPULATION DENSITY

The home addresses of the patients were obtained from the register of patients with cystic fibrosis held at St James's Hospital, and electoral wards for these were then obtained from the voting register held at city libraries and town halls. Having established the electoral ward, the population densities were obtained from census statistics. ${ }^{4}$

\section{Results}

MEASUREMENT OF ANTIBODY TO ASPERGILLUS The study population was divided into two groups with high and low titres of antibody to aspergillus. The value chosen to do this was $21 \%$ SBI. This was the median titre $(2 \%)$ plus two standard deviations $(9.6 \%)$ for the 60 patients in the clinic who had never grown $A$ fumigatus in sputum cultures during the preceding year. There were 98 patients with titres less than $21 \%$ SBI in the low titre group and 39 in the high titre group.

\section{POPULATION DENSITY}

Information on electoral wards was obtained for $135(99 \%)$ of the clinic population. Patients from the group with high titres of antibody to aspergillus lived in less densely populated

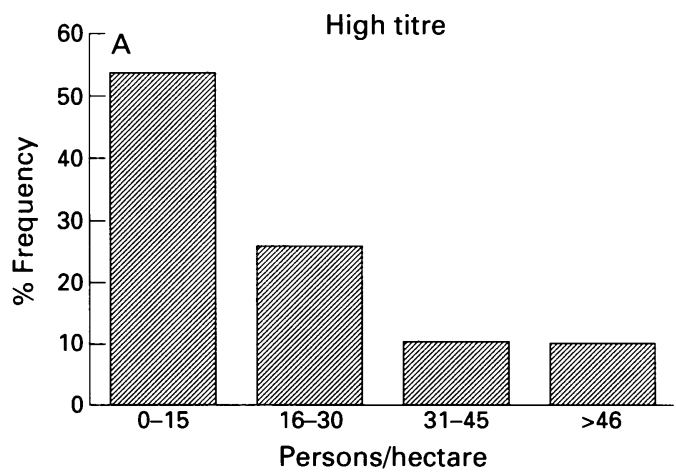
give the sample result.

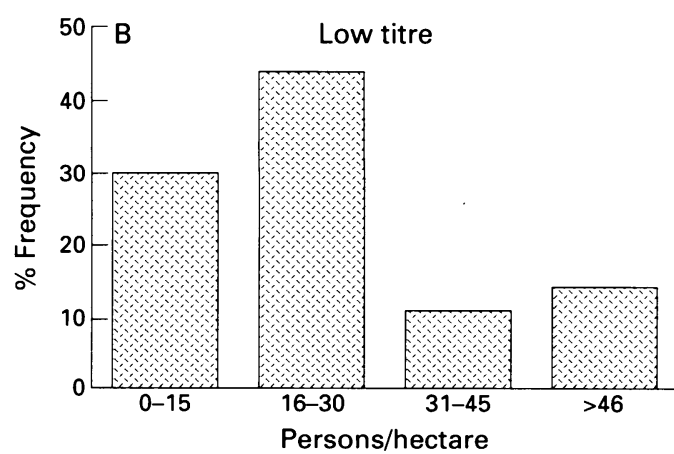

Population density of area of residence for patients with $(A)$ high and $(B)$ low titres of antibody to aspergillus. 
electoral wards than those from the low titre group (figure). The median population density for the high titre group was 12.03 compared with 19.07 people/hectare for the low titre group ( $<<0.02$ Mann-Whitney $U$ test). The proportion of patients from each group living in a ward with a population density of 15 people/hectare or less (that is, a suburban or rural area) was $21 / 39(54 \%)$ from the high titre group compared with $29 / 96(30 \%)$ in the low titre group $\left(\chi^{2}=12 \cdot 8, p<0 \cdot 001\right)$.

\section{Discussion}

Previous investigations of aspergillosis in patients with cystic fibrosis have largely studied the relation between levels of antibody to aspergillus and clinical status. ${ }^{1}$ Other potential associations with antibody response to $A$ fumigatus in patients with cystic fibrosis have not been investigated. We speculated that colonisation with $A$ fumigatus may partly be determined by the amount of exposure to the fungus. We found that a significantly greater proportion of patients with high titres of antibody to aspergillus lived in areas with a lower population density. This suggests that a rural environment is more likely to be associated with the development of colonisation with $A$ fumigatus and the subsequent immune response to the fungus. This may be caused by increased exposure to fungal spores in a rural environment.

A proportion of patients with cystic fibrosis colonised with $A$ fumigatus develop allergic bronchopulmonary aspergillosis (ABPA). ${ }^{5}$ Whether environmental exposure to high spore concentrations is important in the aetiology of
ABPA has not been well studied. There were too few patients with ABPA in this study to show any environmental association, though this is likely as a humoral immune response is necessary to develop the disease. Certainly exposure to known sources of the fungus has been temporally associated with the development of ABPA. For instance, we have noted four cases of the disease in patients with cystic fibrosis who visited horse riding stables shortly before presenting with symptoms. ${ }^{6}$

In conclusion, we have shown an association between a rural environment and high titres of antibody to aspergillus in patients with cystic fibrosis and we speculate that increased exposure to $A$ fumigatus spores is the cause of this. Differences in living environments may explain some of the variation in the incidence of colonisation with $A$ fumigatus and of ABPA reported from different cystic fibrosis clinics.

We thank the Cystic Fibrosis Research Trust and Janssen We thank the Cystic Fibrosis Research Trust and Janssen Pharmaceuticals Limited for providing financial support to

1 Forsyth KD, Hohmann AW, Martin AJ, Bradley J. IgG antibodies to Aspergillus fumigatus in cystic fibrosis: a laboratory correlate of disease activity. Arch Dis Child 1988; 63: 953-7.

2 Marsh PB, Millner PD, Kla JM. A guide to the recent literature on aspergillosis as caused by Aspergillus fumigatus, a fungus frequently found in self-heating organic matter. Mycopathologia 1979; 69: 67-81.

3 Richardson MD, Stubbins JM, Warnock DW. Rapid enzyme-linked immunosorbent assay (ELISA) for Aspergillus fumigatus antibodies. $\mathcal{f}$ Clin Pathol 1982; 35: 1134-7.

4 Office of Population Censuses and Surveys. Census 1981: ward and civil parish monitor. London: HMSO, 1981.

5 Simmonds EJ, Littlewood JM, Evans EGV. Allergic bronchopulmonary aspergillosis and cystic fibrosis. Arch Dis Child 1990; 65: 507-11.

6 Simmonds EJ, Littlewood JM, Evans EGV. Allergic bronchopulmonary aspergillosis [letter]. Lancet 1990; 335: 1229. 